ConNotas. Revista de crítica y teoría literarias / Vol. V, Núm. 9 / 2007

\title{
Desde la Historia -y la historia- hasta la identidad política: estrategia narrativa del General Vicente Riva Palacio en Monja y casada y Martín Garatuza
}

\author{
Yun SOOK KIM*
}

\section{Resumen:}

Luego de que México lograra independizarse, fue necesario conformar en la conciencia del pueblo la imagen de una nación que, al margen de las herencias de la Colonia, adquiriera identidad autónoma. De este modo, a la lucha de pólvora y cañones le sucedió una lucha cuya arma principal fue la pluma de los escritores. Así, en una situación liminal en la que el paradigma colonial estaba derrumbándose y el nuevo concepto de nación sólo era la firma sobre un papel, Ignacio Manuel Altamirano (18341893) propone a la novela histórica como el conducto a través del cual se cristalizaría la nueva conciencia nacionalista. Vicente Riva Palacio (1832-1896), uno de los principales seguidores de la doctrina de Altamirano, lleva a cabo la propuesta de este último cuando escribe dos de sus novelas: Martín Garatuza (1868) y Monja y casada, virgen y mártir (1868). En ambas novelas, detrás de una trama de corte dramático, e incluso romántico, Riva Palacio supo esconder un mensaje para el lector de la época: a saber, que era imprescindible superar los lineamientos de la Colonia y mirar más atrás, al instante primordial, donde se encontrarían los elementos para construir la nueva identidad de la nación. 
Palabras clave:

Ignacio Manuel Altamirano, nacionalismo, paradigma cultural, conciencia colonial, novela histórica, pedagogía.

Cuando Benito Juárez subió al poder por segunda vez, en 1868, México estaba profundamente dividido políticamente. Juárez se enfrenta a la tarea de restaurar la República constitucional que el clero y los conservadores habían atacado directamente con ejércitos propios y con invasores extranjeros. Juárez pone en vigencia legal las leyes de Reforma que buscan instaurar plenamente por lo menos los tres principios liberales clásicos de la igualdad, la libertad, y la fraternidad, con la intención de acabar con la desigualdad política, económica y social que aquejaba a la Nación y al Estado. Busca Juárez adelantar esta labor merced a una política conciliadora, bajo su bien conocido dictado de que "el respeto al derecho ajeno es la paz".

Esta política restauradora y conciliadora se vio apoyada, en el campo de la cultura y de las letras, principalmente por dos figuras que habían luchado con las armas junto al mandatario, en los campos de batalla. Fueron ellas Ignacio Manuel Altamirano, y Vicente Riva Palacio. Ambos contribuyeron a la creación de un espíritu de unión entre los mexicanos.

El Maestro Altamirano estableció su tribuna desde su periódico El Renacimiento, y a partir de las veladas literarias que propició. Se dio cuenta de la importancia de la pedagogía, y de una nueva pedagogía que privilegiara los valores nacionales, de un nacionalismo cultural, que habría de manifestarse en el estudio de la sociedad nacional, pasada y presente, como elemento activo de la integración ciudadana. José Luis Martínez nos recuerda que, en El Renacimiento, Altamirano estimuló la publicación de escritos mexicanos, ya sobre la historia y la crítica de poesía, de literatura nacional en general, sobre fechas y efemérides mexicanas o disertaciones sobre bibliología tocantes a asuntos mexicanos, buscando "atraer hacia lo nacional tanto a los hombres de estudio como a los artistas [...]" (Expresión 105). 
Este nacionalismo cultural pronto se convierte en historicismo, en lo que podríamos llamar el segundo historicismo mexicano, que predicaba una mirada hacia atrás, para hacer ver lo grande y ejemplar en los monumentos de cultura del país, en los hombres heroicos en que abunda su historia, y en la historia de sus ideas altruistas y patrióticas, sin olvidar a los enemigos de las mismas. Escribió, a propósito:

La historia antigua de México es una mina inagotable [...] las grandiosas ruinas de Yucatán, de Palenque y de Puebla [...] Las páginas de Gómara, de Ixtlilxóchtl y de Clavijero [...] Pero estos tesoros a nadie deben enriquecer más que a los historiadores mexicanos [...] Ahí está esa Inquisición terrible, que viene también de Europa pretendiendo 'quemar las ideas' en América [...]. ("Revistas" 34)

El historicismo pronto se convierte en historicismo político: se trataba de recordar para cuestionar la interpretación histórica tradicional, particularmente la visión de Lucas Alamán. Se propone una revisión de la historia con ojos renacidos, en forma que el espíritu de unión patriótica con los que eran enemigos antes no significaba olvido, ni aceptación de la interpretación histórica tradicional y conservadora. La generación de la Reforma y de la República Restaurada miraba hacia atrás, en momentos en que se empeñaba en mirar hacia delante, en construir un nuevo país, precisamente porque el recuerdo, es decir, la historia, está unida al deseo, es decir, la Utopía, según bien lo dice Carlos Fuentes (Valiente 48). Para construir, muchas veces hay que destruir. Desde el poder que tienen ahora los constructores de la República, estaban empeñados en borrar la herencia horrenda de la Colonia aún presente ${ }^{1}$ en la sociedad de

${ }^{1}$ Leticia Algaba Martínez dice que en el siglo XIX el orden colonial aún es defendido por los conservadores en México y que las Leyes de Reforma contrarrestan este orden de cosas (Licencias 68). La misma autora se hace la pregunta que se hacen los Reformadores, según ella: “¿Cuáles sucesos del pasado inciden y cobran vigencia en el presente?" (99). 
la época. A su vez, el llamado al nacionalismo historicista de Altamirano pronto se convierte en un llamado al nacionalismo historicista literario, a propósito de lo cual declara:

[...] la juventud de hoy, nacida en medio de la guerra y aleccionada por lo que ha visto, no se propone sujetarse a un nuevo silencio. Tiene el propósito firme de trabajar constantemente hasta llevar a cabo la creación y el desarrollo de la literatura nacional. [subrayado nuestro] ("Revistas" 33).

La guerra mueve a los jóvenes a no callarse, como antes y, por el contrario, a recordar las experiencias históricas. La mención de los temas históricos dignos de tratar, entre ellos el de "esa Inquisición terrible que viene también de Europa pretendiendo 'quemar las ideas' de América" ("Revistas" 34), es una muestra de cuál es el historicismo que plantea el Maestro. El Maestro concreta su llamado a hacer historicismo literario cuando llama a hacer novela historicista, y cuando, a la vez, la une a la labor pedagógica en que estaba empeñado el nuevo régimen liberal. Se da cuenta de que la novela es el género de mayor acogida popular, y la propone entonces como el género más a propósito para llevar adelante la labor pedagógica de las nuevas doctrinas entre los lectores:

La novela es indudablemente la producción literaria que se ve con más gusto por el público, y cuya lectura se hace hoy más popular. [Es...] el artificio con que los hombres pensadores de nuestra época han logrado hacer descender a las masas doctrinas y opiniones que de otro modo habría sido difícil hacer que aceptaran. ("Revistas" 39)

En este momento conviene observar hacia dónde apunta la aparente estrategia del estratega-en-jefe del programa cultural de la República Restaurada, el Maestro Altamirano, para observar luego cómo la estrategia literaria del más importante de sus discípulos la lleva adelante. En efecto, y en resumen, Altamirano llama a un nacionalismo historicista que, entre otras manifestaciones se exprese en lo pedagógico de la nueva doctrina política y en lo literario, par- 
ticularmente en el género novelesco. Equivale esto a decir que esta manifestación apunta en últimas a tener un efecto en las masas mexicanas de lectores, en la comunidad imaginaria de lectores. Es por esto que se ocupa, en últimas de la relación novelista-lector, y en dar orientaciones para uno y para otro miembro de esta dualidad, a fin de asegurar la unidad de esa imaginaria comunidad lecto$\mathrm{ra}$, alrededor de un nuevo mensaje. La verdad es que si se querían cambiar las prácticas tradicionales del dominio de una minoría hegemónica conservadora, monárquica, autoritaria, apátrida y privilegiada, liderada por la Iglesia, había que reinterpretar la visión histórica que esa minoría había instilado en la mente de los mexicanos, estableciendo una nueva pedagogía que uniera a las masas -a la democracia, a las mayorías- y que les diera poder y las identificara en un nuevo propósito nacional -o Renacimiento-, dentro del ideario de la democracia liberal.

El Maestro propone normas para la escritura y normas para la lectura de esa nueva novela. Propone una forma de codificación y la correspondiente forma complementaria y recíproca de decodificación de la misma. Se trata de la escritura de una novela, pero no una novela histórica cualquiera, o parecida a la de la tradición. Habrá de ser una novela en la que el novelista aúne la visión Histórica dentro de su historia (story) particular, a lo político, lo social, lo moral, y cuyo mensaje deberá ser descubierto por el lector avisado, en una especie de juego complementario y recíproco de esconderencontrar, cuando dice:

[...] la novela hoy ocupa un rango superior, y aunque revestida con las galas y atractivos de la fantasía, es necesario no confundirla con la leyenda antigua, es necesario apartar sus disfraces y buscar en el fondo de ella el hecho histórico, el estudio moral, la doctrina política, el estudio social, la predicación de un partido [...] en fin, una intención profundamente filosófica y trascendental en las sociedades modernas. La novela hoy suele ocultar la biblia de un nuevo apóstol, o el programa de un audaz revolucionario. ("Revistas" 39) 
Nadie mejor calificado para llevar a cabo esa estrategia de 'busca y esconde' (seek and hide) que un periodista político mexicano como Vicente Riva Palacio, un estratega militar y un prolífico y notable escritor. Vicente Riva Palacio fue el apóstol del nuevo credo, anunciado por Altamirano. De hecho, Altamirano lo nombra como miembro destacado de la nueva escuela de la novelística nacional cuando dice explícitamente: "En la nueva escuela que se ha reunido hay soldados de la República como Riva Palacio, que acaban de desceñirse la espada victoriosa [que son miembros de] este renacimiento literario" ("Revistas" 34). Riva Palacio, como novelista, es discípulo de las ideas de Altamirano, a que nos hemos referido, y comprende que el escritor es un maestro de una nueva pedagogía, ${ }^{2}$ que hay que escribir una nueva novela nacionalista, que debe ser histórica, que no debe ser un mero cuento estúpido y sentimentalista, que debe ser una Biblia que contenga "el programa de un audaz revolucionario"; se da cuenta, además, de que la Historia, como lo dice David Maciel, empieza a utilizarse -por los Restauradores- como arma de Estado, y para "fortalecer la lealtad del ciudadano a su patria [...]" (Ignacio 131), es decir, para fortalecer la identidad del lector mexicano con la ideología del Estado liberal democrático.

Terminada la guerra contra la Intervención francesa, Riva Palacio entrega el mando militar de Michoacán y decide retirarse al cultivo de las letras. A la novela histórica dedicó gran parte de su empeño, con dos de las cuales que se destacan al respecto, a saber, Monja y casada, virgen y mártir; historia de los tiempos de la Inquisición, publicada por entregas en La Orquesta, en 1868, y Martín Garatura; memorias de la Inquisición, publicada en el mismo periódico, a continuación, y en cierta forma como continuación de la anterior, también en $1868 .^{3}$

${ }^{2}$ Riva Palacio nos comunica su deseo de instruir deleitando -como otro Horacio-, en todo de acuerdo con Altamirano, y se refiere a sus novelas como libros que "en todo caso prestan el insigne servicio de popularizar los conocimientos [...] evitando el escollo del fastidio: tal es mi deseo" (Memorias, "Prólogo" viii).

${ }^{3}$ Otras novelas históricas del General Vicente Riva Palacio son, Calvario y Tabor; novela historica y de costumbres, publicada en 1868, Las dos emparedadas: memorias de la 
Libradas las batallas por la Reforma con la espada, el General se dedica a ganar otras batallas con la pluma, y a formar batallones de lectores convencidos, defensores del nuevo credo.

Escondido en el subtexto de las dos mencionadas novelas, mediante un juego alegórico y connotativo, está el mensaje para los lectores contemporáneos: la sociedad colonial cuya estructura y prácticas sociales retrógradas y anacrónicas pertenecientes a la Colonia se describen en ellas, está aún viva y vigente en su tiempo; contra tales estructuras y prácticas se alza la causa de la Restauración republicana, con la que hay que identificarse en este momento de encrucijada crítica entre el pasado y el porvenir.

Detengámonos unas líneas para examinar las características de la mencionada estrategia del general-novelista y su efecto en el lector, así como la forma en que el lector lee las dos novelas y, sobre todo, finalmente, para qué usa Riva Palacio esa estrategia.

Riva Palacio apela a un juego sucesivo de alegoría $\rightarrow$ empatíasimpatía $\rightarrow$ alegoría, según lo veremos a continuación. Merced a ese juego literario busca la unión entre su numeroso público lector alrededor de las ideas de la República Restaurada, y como consecuencia, la identificación de la comunidad lectora dentro del nuevo Estado propuesto por las mismas.

La estrategia o juego empleado por el general-escritor conduce desde la Historia y la story hasta la identificación de la comunidad lectora alrededor de las ideas de la Reforma, y comprende los siguientes pasos: primero, identificación alegórica entre protagonistas de la historia narrada (story) con los héroes de la Historia mexi-

Inquisición, publicada en 1869, Los piratas del golfo; novela histórica, publicada en 1869 , La vuelta de los muertos; novela histórica, publicada en 1870, y Memorias de un impostor: Don Guillén de Lampart, rey de México; novela histórica, publicada en 1872. De carácter histórico son los capítulos que escribió en el Libro rojo, en 1871, así como Historia de la administración de D. Sebastián Lerdo de Tejada, aparecida en 1875, y el volumen segundo que con el título de "El Virreinato; Historia de la dominación española desde 1521 a 1808" se publicó en la obra colectiva titulada México a través de los siglos, aparecida entre 1884 y 1889. Waal, Fans de. Our Inner Ape. New York: Riverhead Books, 2005. 
cana; segundo, identificación empática y simpática entre los lectores y los protagonistas de las dos novelas; tercero, descubrimiento, por parte del lector, de que el medio social en que se debaten los protagonistas de la ficción presentada en las dos novelas y los agonistas de la Historia de México son alegoría del medio social en el que se debate él mismo; cuarto, en consecuencia, unión e identidad de los miembros de la comunidad lectora de Riva Palacio, alrededor del deseo de un nuevo Estado, precisamente el proclamado por la Reforma.

En el primer paso, los protagonistas de las dos novelas se identifican con los héroes de la Historia mexicana, en parte, genealógicamente, como sucede en Martín Garatuza, y en parte, alegóricamente, como sucede tanto en Martín Garatuza como en Monja y casada. En efecto, los miembros de la familia protagonista Carvajal-Salazar en Martín Garatuza se identifican con el héroe por antonomasia de México, Cuauhtémoc por sangre -indígena- y por ideas -resistentes contra el régimen español- en el curso de cuatro generaciones constituidas por Felipe, el mestizo hijo de Cuauhtémoc y sus hijas, Isabel, Violante y Leonor, así como Juana, hija de Isabel, y Esperanza, hija de Juana, y Leonel, hijo de la hermana de Juana; todos se identifican con el héroe en cuanto reciben su gota de sangre indígena y la herencia de su credo político sintetizado en las palabras "[...] mi imperio volverá a ser Uno y solo, y Tenochtitlán será libre [...]", pronunciadas por el héroe (Martín 1: 170).

Al mismo tiempo, tanto los protagonistas de Martín Garatuza como los de Monja y casada, se identifican alegóricamente con los héroes de la Historia de México. Los héroes y personajes históricos con los que se identifican los protagonistas de ambas novelas son: de nuevo Cuauhtémoc, así como la familia Carabajal, verdaderamente procesada, atormentada y quemada por la Inquisición (proceso cumplido entre 1587, 1598, y 1601), Sor Blanca del Corazón de Jesús, Sor Juana Inés de la Cruz, Josefa Ortiz de Domínguez, Miguel Hidalgo y Costilla, y los insurgentes Vicente Ramón Guerrero Saldaña, y José Maria Morelos, todos ellos personajes históricos pertenecientes a la resistencia activa, ya intelectual, ya armada, contra el Estado colonial. 
Con Cuauhtémoc, el héroe torturado, cuyos pies fueron quemados y cuyo fin fue la horca, se asocian alegóricamente las vidas, los padecimientos y la muerte de gran parte de sus descendientes novelescos, particularmente su hijo Felipe y sus tres hijas, procesadas y quemadas injustamente por la Inquisición, según las páginas de Martín Garatuza.

Con la familia Carabajal histórica, particularmente con las mujeres (doña Francisca Núñez y sus hijas doña Isabel, doña Leonor, doña Catalina, y doña Mariana) se identifican alegóricamente Blanca y Luisa, protagonistas de Monja y casada. La persecución y terribles torturas a que fueron sometidas ambas en las cámaras de tormento de la Inquisición se describen en la novela en forma semejante a como se describen las que sufrieron doña Francisca Núñez de Carabajal y su hija mayor doña Isabel, según se contienen en documentos históricos. Con las mujeres de la familia Carabajal histórica se identifican alegóricamente también las protagonistas Isabel, Leonor, y Violante, hijas de Felipe, muertas en el Auto de Fe que se narra en Martin Garatuza. Esta narración es semejante al testimonio histórico recogido por Riva Palacio en El libro rojo (265318 ), y la que Genaro García y Carlos Pereyra han recogido en fiel documentación histórica (50-56).

La Blanca Mejía de Monja y casada se identifica alegóricamente con Sor Blanca del Corazón de Jesús, monja profesa del Convento de Santa Teresa de las Carmelitas Descalzas, llamada antes de entrar al convento Blanca de Mejía. El Edicto del Santo Oficio contra Sor Blanca, perseguida como la Blanca novelesca por el Tribunal, por haber huido del convento como ésta, se publica en la novela mencionada.

Doña Juana, la madre de Esperanza, en Martín Garatuza se identifica alegóricamente con Sor Juana Inés de la Cruz, particularmente por sus intereses intelectuales y su posición rebelde. También por ser autora de libros, en este caso, unas Memorias familiares. También se identifica alegóricamente doña Juana con Josefina Ortiz de Domínguez, "La Corregidora”, mujer que desempeñó, como se sabe, papel importantísimo en el levantamiento de Miguel Hidalgo y Costilla: ambas participan en reuniones de complotados contra el régi- 
men español, y ambas fueron consejeras del grupo de patriotas que pensaban dar el grito de independencia, tanto en la realidad, como en la obra.

El padre Alfonso Salazar, uno de los protagonistas principales de Martín Garatuza se identifica alegóricamente tanto con el padre Hidalgo, en cuanto cura y en cuanto dirige, como éste, un grupo de conspiradores patriotas preindependentistas, como con el padre José María Morelos, por miembro de la insurgencia y por descendiente, como éste, de uno de los gobernantes aztecas. En fin, Leonel, el protagonista de Martín Garatuza, se identifica tanto con Hidalgo como con Morelos, en cuanto jefe del grupo de patriotas que en la obra piensan participar en la insurrección de independencia, y con Vicente Guerrero, por ser descendiente de uno de los reyes del Imperio Azteca.

En el segundo paso el escritor busca que el lector/lectora se identifiquen con la heroína/héroe de las dos novelas, mediante la lectura empática/simpática a que éstas se prestan. Hay que tener en cuenta que en el siglo XIX, los lectores estaban enamorándose de las hermosas heroínas y, a su vez, las lectoras estaban haciendo lo mismo con los apuestos galanes literarios. Doris Sommer habla del "broad appeal of the power that enthralled readers who wanted to possess or to be possessed by the heroes and heroines of novels" de los nuevos países que se estaban estructurando en la América Latina, durante el siglo XIX (Foundational 34). El fenómeno es empático, y la empatía "prompts forward an identity beyond relationship, a common identity that enhances the separate identities of individual persons" (Morrison xxvi) y hace que "Yo" sea "Tú" (Adam Smith citado por Waal 2), y que tu pena sea mi pena o pena compartida, que es lo que significa la palabra 'simpatía'. Al identificarse empática/simpáticamente el lector/lectora con la heroína/ héroe, llegan a compartir sus deseos amorosos y a desear unos y otros un final feliz para la historia contada, es decir, la desaparición de los obstáculos que separan a los amantes novelescos. Pero en las novelas fundacionales, como lo son, según nosotros, Monja y casada y Martín Garatuza, los obstáculos no son de orden personal, sino políticos y sociales. En estas dos novelas, así como en las que exa- 
mina Doris Sommer "These obstacles are [...] a social convention or a political impasse; that is, they are public and interpersonal rather than some intimate and particular difference between the lovers" (Foundational 48). Y si, como en el caso de Monja y casada y Martín Garatuza, el obstáculo es de naturaleza social y política, impuesto por el Estado monárquico, entonces “it [...] heightens their/our [de los lectores] love for the possible nation in which their affair could be consumated" (subrayado nuestro) (Foundational 48), ["it heightens the love for] the new state that would joint them" (Foundational 32), es decir, aumenta entre personajes y lectores el deseo de un Estado republicano y antimonárquico en el cual pueda ser posible la unión de los amantes Blanca y César (de Monja y casada) y Leonel y Esperanza (de Martín Garatuza), y el deseo de la desaparición de los obstáculos que los separan. ¿Y cuáles son esos obstáculos que se interponen entre las dos parejas de amantes que amantes y lectores quieren que desaparezcan?

Riva Palacio presenta la serie de obstáculos: el ejército colonial, la Iglesia, la Inquisición, la burocracia española gobernante, la elite económica colonial, organizaciones corruptas y criminales. En Monja y casada, Blanca -la novia de César- es forzada a entrar en un convento, por un lado, por su hermano Pedro de Mejía, "el hombre más rico de México", quien espera poder robarle, merced a esta argucia, la parte de la herencia familiar que le pertenece, y por otro lado, por Beatriz, la fanática religiosa madrina suya. Han conseguido, antes, separarla de César -a quien hacen enviar a Filipinas-, a fin de que no casándose, Blanca no pueda heredar. El malvado Pedro de Mejía vuelve a aparecer casi al final de la obra, cuando le paga a la Inquisición una buena "limosna" para que mate a su hermana, que a la sazón ha sido encarcelada y torturada por ese Tribunal. La Inquisición se encarga de hacer de Blanca una mártir -como se deja ver en el título de la novela- para castigar el "pecado" cometido al huir del convento en busca de su libertad y su destino de mujer en la sociedad, como esposa. Para castigar el presunto delito de salirse de un convento donde había sido encerrada de por vida contra su voluntad, y buscar el libre ejercicio de su personalidad, la Inquisición la persigue en la forma más encarnizada, la apresa y la tortura, 
y dispone matarla en la hoguera. Es decir, el malvado hermano se alía con la Iglesia y la Inquisición para separar a los amantes, y arruinar sus vidas, en beneficio económico de los dos aliados, Mejía y la Inquisición.

En Martín Garatuqa, la unión entre los novios Leonel y Esperanza, retoño último de la familia Carvajal-Salazar que tiene su origen en Cuauhtémoc, ha sido amenzada durante cuatro generaciones, tanto por el ejército español como por la Inquisición y los estamentos de la sociedad discriminante peninsular. En efecto, el ejército de Cortés corta con la muerte de Cuauhtémoc su relación amorosa con la española Isabel, aunque no pudo impedir que de la misma naciera el mestizo Felipe, primer vástago de la familia. Felipe engendra tres hijas -doña Violante, doña Leonor, y doña Leonor de Carvajal- pero la mala fortuna hace que Baltasar Salmerón, miembro de la clase española gobernante, se enamore de Isabel. Esta no le corresponde y se casa con su primo Nuño de Carvajal. El enardecido Baltasar Salmerón la rapta y la viola, y para vengarse añadiendo injuria al insulto, mata a escondidas a su marido, y denuncia a todas las hermanas ante la Inquisición como judaizantes. Como resultado, las tres hermanas y Felipe son apresados y atormentados por la Inquisición, las tres hermanas son quemadas públicamente, y las posesiones de la familia son robadas por el Tribunal. En este caso, la criminal complicidad con Salmerón -a quien la justicia no persigue- le ha reportado jugosos beneficios económicos a la Inquisición. De esta debacle se ha salvado, sin embargo, Juana, hija de Isabel y de Nuño, y continuadora de la cadena familiar que llega hasta su hija, doña Esperanza la novia, protagonista de la obra.

El noviazgo entre Esperanza y Leonel ha sido doblemente amenazado: por un lado, como se ve, por el peligro en que en que se vieron los miembros de cada eslabón de la cadena que conduce desde Cuauhtémoc hasta ella, causado por el ejército español, los malvados miembros de la elite peninsular y la Inquisición -metonimia de la Iglesia- ladrona, fanatizadora, enemiga de la libertad de conciencia, de la libertad de la mujer, torturadora y asesina, en tanto que por otro lado, tal noviazgo ha sido amenazado también por los peligros puestos ante él y ella, por la discriminación existente 
entre criollos y peninsulares, entre descendientes de judaizantes y cristianos.

En Martín Garatuza, Leonel está enamorado de Esperanza, y ésta le corresponde con amor. Sin embargo, la discriminación y persecución que la administración española ejercía contra los criollos novohispanos se interpone como un primer obstáculo entre la pareja de novios. En efecto, Esperanza, contra sus sentimientos, dice que no podrá ni querrá casarse con Leonel, porque no quiere tener hijos con la mancha de criollos, en los cuales se perpetúe la humillación que tales criollos sufren en el país. Además, Esperanza es descendiente de una familia manchada con el estigma de judaizantes, es decir, con la mancha social que la Inquisición ha usado para perseguir, con pretextos religiosos, a quienes se oponen al régimen.

El padre de Leonel, Nuño de Salazar, español arrogante, odia a la familia de Esperanza -a pesar de tener nexos familiares con ellos-, por ser criollos que están contra el régimen monárquico. Trata de impedir la relación amorosa y le dice: “-[...] no me gusta que frecuentes amistades de esa clase [... tu madre] era prima de doña Juana [la madre de Esperanza] al fin era criolla y tenía tales relaciones; pero en lo sucesivo, ese parentesco como si no existiera" (Martín 1: 20-21). Y en algún momento llega hasta a manifestar que se arrepiente de ser padre tanto de Leonel como de su hermano, el cura Alfonso, al saber que también son conspiradores.

En suma, los obstáculos que se presentan ante los amantes hacen desear a los lectores/lectoras, que desaparezcan los obstáculos que se interponen o imposibilitan la realización de su unión, que en definitiva son de orden político, pues están engranados en la naturaleza del Estado español colonial. Es decir, los lectores llegan con ello a querer, tanto como los protagonistas, la desaparición del régimen político colonial español en Nueva España, lo que equivale a desear un nuevo Estado en el que la unión matrimonial pueda realizarse. Con esto llegan a desear lo mismo que desearon, en lo político, los héroes progresistas de la Historia, y lo mismo que desean los protagonistas de las dos obras, a saber, el Estado republicano.

Es este el momento en el que como epifanía se da el paso tercero, como efecto, en la mente del lector avisado, aquél que ha acata- 
do la norma de lectura propuesta para la nueva novela histórica por el Maestro Altamirano. El lector descubre que Riva Palacio ha retratado en el medio social en el que se debaten los protagonistas de las dos novelas, y los héroes de la Historia, muchas de las características del medio social en que se debate el lector mismo. Para el caso del lector mexicano de la época de Riva Palacio, esas características tienen que ver con las estructuras y prácticas sociales retrógradas y anacrónicas pertenecientes a la Colonia, y por lo tanto ya condenables en la nueva época. En efecto, en retratar la sociedad del lector en la sociedad de los protagonistas de la story y de la Historia, consiste la construcción alegórica última hecha por el autor, que así cumple, también, con la norma de escritura de la novela histórica propuesta por Altamirano. Se cumple así, también, tanto en Monja y casada, como en Martín Garatuza, con la característica alegórica que los textos "Tercermundistas" tienen, según Frederic Jameson, quien sobre ellos dice, al respecto:

All third-world texts are necessarily, I want to argue, allegorical, and in a very specific way: they are to be read as what I will call national allegories", "[...] Third-world texts, even those which are seemingly private and invested with a properly libidinal dynamic necessarily project a political dimension in the form of national allegory: the story of the private individual destiny is always an allegory of the embattled situation of the public third-world culture and society. ("Third-World" 69)

Las dos obras están, pues, escritas de manera que el lector las lea como alegorías de los abusos de la sociedad en la que el lector vive, y que aún conservaba muchos rezagos de la sociedad monárquica y autoritaria de la Colonia (Algaba 99). Para que leyera en la story la Historia actual suya, y para que deseara ya no sólo la desaparición de los abusos y atrocidades que sufrieron los protagonistas de la ficción y los agonistas de la Historia pasada, sino -más importante aún- para que deseara la desaparición de los abusos con los que instituciones y prácticas retrógradas (Iglesia, conventos, espíritu inquisitorial religioso, intromisión de la Iglesia en los asuntos del 
Estado, ejército tradicional, tendencias monárquicas) le hieren en la vida práctica cotidiana, y luchara políticamente para lograr ese deseo.

El cuarto paso estratégico es una consecuencia del anterior. Riva Palacio estaba escribiendo novelas que permitían no sólo la identificación individual del lector con personajes -novelescos e Históricos- victimados, sino que también permitían que los lectores se identificaran entre sí como miembros de un mismo grupo político que era víctima de un mismo autoritarismo y (neo) colonialismo, esta vez dentro de la sociedad postindependentista de México. Crea de esta manera una comunidad imaginaria de lectores que se identifican colectivamente con su Historia (por mediación de las ficciones novelescas) y que están dispuestos, como ejército de civiles, a decir 'no' a la reacción retrógrada que pretendió atosigarlos. Ha contribuido el General a la creación de un ejército de corazones y mentes convencidos en los principios de la Reforma, para la paz y la civilidad, como antes los había creado al organizar ejércitos de soldados para la guerra y la milicia, en la defensa de los mismos principios. Ejércitos de civiles que creen en el nexo entre su presente y el futuro utópico. Ha contribuido a transformar la historia de ficción (story) y la Historia, en identidad.

\section{Bibliografía}

Algaba Martínez, Leticia. Las licencias del novelista y las máscaras del crítico. México: Universidad Autónoma Metropolitana Azcapotzalco, 1997.

Altamirano, Ignacio Manuel. "Revistas literarias de México (18211867); primera revista." Ignacio Manuel Altamirano. Obras completas (Escritos de literatura y arte). Vol. 12, tom. 1. México: Secretaría de Educación Pública, 1988.

Fuentes, Carlos. Valiente mundo nuevo; épica, utopia y mito en la novela hispanoamericana. Madrid: Mondadori España, 1990.

García, Genaro y Carlos Pereyra. Documentos inéditos o muy raros para la historia de México. México: Librería de la Vda. De Bouret, 1906. 
Jameson, Fredric. "Third-World Literature in the Era of Multinational Capitalism." Social Text 15 (Fall 1986): 65-88.

Maciel, David R. Ignacio Ramírez, ideólogo del liberalismo social en México. México: Universidad Autónoma de México, 1980.

Martínez, José Luis. La expresión nacional; letras mexicanas del siglo XIX. México: Imprenta Universitaria, 1955.

Morrison Kart Frederick. I am You: the Hermeneutics of Empatby in Western Literature, Theology, and Art. Princeton, N. J.: Princeton UP, 1988.

Riva Palacio, Vicente. "La familia Carabajal." Primera parte. Vicente Riva Palacio, Manuel Payno, Juan A. Mateos, y Rafael Martínez de la Torre. El libro rojo; 1520-1867. 1871. Vol. 1. México: A. Pola, 1905. 265-291; 292-317; 318-350.

Martín Garatuza. 1868. 2 vols. $3^{\text {a }}$ ed. Ed. y pról. Antonio Castro Leal. México: Porrúa, 1975.

Memorias de un impostor; don Guillén de Lampart, rey de México. México: Manuel C. de Villegas, 1872.

Monja y casada, virgen y mártir. 1868. 2 vols. $3^{a}$ ed. Ed. y pról. Antonio Castro Leal. México: Porrúa, 1974.

Sommer, Doris. Foundational Fictions: the National Romances of Latin America. Berkeley, Los Angeles, y London: U California P, 1993. Waal, Fans de. Our Inner Ape. New York: Riverhead Books, 2005. 